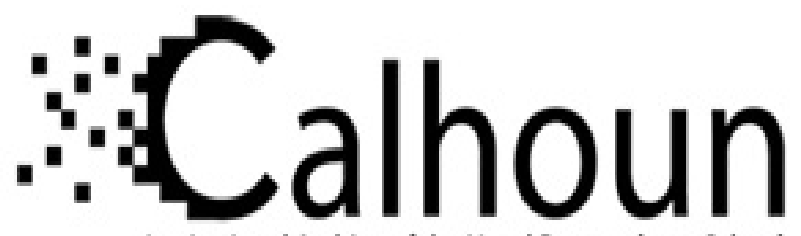

Institutional Archive of the Naval Postgraduate School

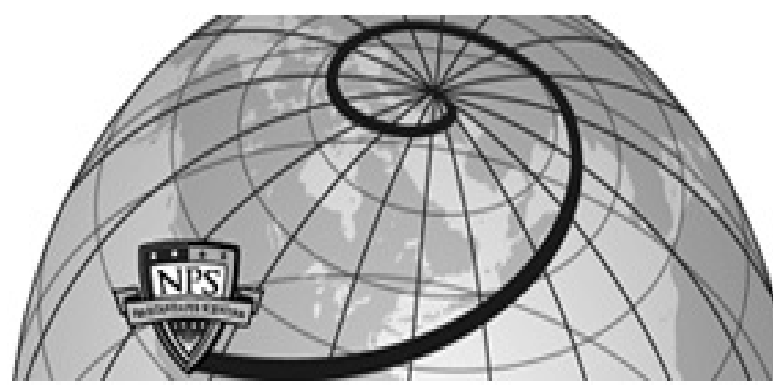

Calhoun: The NPS Institutional Archive DSpace Repository

Analysis of Imaging Spectrometer Data Using $\mathrm{N}$-Dimensional Geometry and a Mixture-Tuned Matching Filtering Approach

Boardman, Joseph W.; Kruse, Fred A.

IEEE Transactions on Geoscience and Remote Sensing, Volume 49, No. 11 , November 2011

https://hdl.handle.net/10945/44065

This publication is a work of the U.S. Government as defined in Title 17, United States Code, Section 101. Copyright protection is not available for this work in the United States.

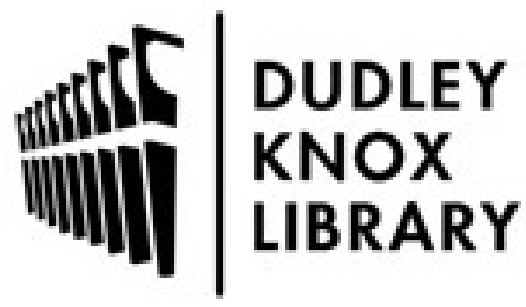

http://www.nps.edu/library
Calhoun is the Naval Postgraduate School's public access digital repository for research materials and institutional publications created by the NPS community. Calhoun is named for Professor of Mathematics Guy K. Calhoun, NPS's first appointed -- and published -- scholarly author.

Dudley Knox Library / Naval Postgraduate School 411 Dyer Road / 1 University Circle Monterey, California USA 93943 


\title{
Analysis of Imaging Spectrometer Data Using $N$-Dimensional Geometry and a Mixture-Tuned Matched Filtering Approach
}

\author{
Joseph W. Boardman and Fred A. Kruse, Member, IEEE
}

\begin{abstract}
Imaging spectrometers collect unique data sets that are simultaneously a stack of spectral images and a spectrum for each image pixel. While these data can be analyzed using approaches designed for multispectral images, or alternatively by looking at individual spectra, neither of these takes full advantage of the dimensionality of the data. Imaging spectrometer spectral radiance data or derived apparent surface reflectance data can be cast as a scattering of points in an $\boldsymbol{n}$-dimensional Euclidean space, where $n$ is the number of spectral channels and all axes of the $n$-space are mutually orthogonal. Every pixel in the data set then has a point associated with it in the $n-d$ space, with its Cartesian coordinates defined by the values in each spectral channel. Given $\boldsymbol{n}$-dimensional data, convex and affine geometry concepts can be used to identify the purest pixels in a given scene (the "endmembers"). $N$-dimensional visualization techniques permit human interpretation of all spectral information of all image pixels simultaneously and projection of the endmembers back to their locations in the imagery and to their spectral signatures. Once specific spectral endmembers are defined, partial linear unmixing (mixture-tuned matched filtering or "MTMF") can be used to spectrally unmix the data and to accurately map the apparent abundance of a known target material in the presence of a composite background. MTMF incorporates the best attributes of matched filtering but extends that technique using the linear mixed-pixel model, thus leading to high selectivity between similar materials and minimizing classification and mapping errors for analysis of imaging spectrometer data.
\end{abstract}

Index Terms-Convex geometry, imaging spectrometry, mixture-tuned matched filtering (MTMF), N-dimensional geometry, spectral endmembers, spectral hourglass, spectral mixing.

\section{INTRODUCTION-IMAGING SPECTROMETRY AND SPECTRAL Mixing CONCEPTS}

$\mathbf{I}$ MAGING spectrometry (simultaneous measurement of continuous spectra and images in up to hundreds of spectral channels or bands) is a proven technology for identifying and mapping materials based on their spectral signatures [1]-[3].

Manuscript received October 31, 2010; revised April 14, 2011 and June 10, 2011; accepted June 11, 2011. Date of publication August 15, 2011; date of current version October 28, 2011. This work was supported in part by the U.S. Army Topographic Engineering Center (now the Army Geospatial Center) under contract DACA76-96-C-0003. Selected algorithms have been implemented in the software package ENVI, which is a commercial product offered by ITT Visual Solutions, Boulder, Colorado.

J. W. Boardman is with Analytical Imaging and Geophysics, LLC, Boulder, CO 80303 USA (e-mail: boardman@aigllc.com).

F. A. Kruse is with the Physics Department and Remote Sensing Center, Naval Postgraduate School, Monterey, CA 93943 USA (e-mail: fakruse@ nps.edu).

Digital Object Identifier 10.1109/TGRS.2011.2161585 (a)

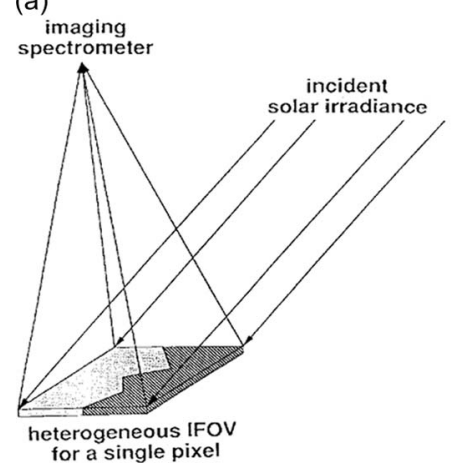

(b) Linear Spectral Mixing for a Single Pixel

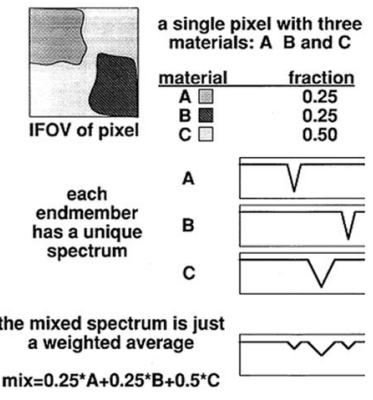

Fig. 1. (a) Two endmember concept of macroscopic (linear) mixing. (b) Three endmember linear spectral mixing showing spatial and spectral context.

It has also become known as "hyperspectral remote sensing," "hyperspectral imaging," and "hyperspectral imagery" or "HSI." Spectral mapping using imaging spectrometer data is well established and routinely used for numerous applications [4]-[10]. These studies, however, indicate that natural surfaces are rarely composed of a single uniform material. Areal mixing within the ground instantaneous field of view of image pixels results in mixed spectral signatures ("spectral mixing" [11]-[13]). Spectral mixing is a consequence of the mixing of materials having different spectral properties within a single image pixel as measured by the imaging spectrometer system (Fig. 1). Several researchers have investigated mixing scales and linearity. Singer and McCord [14] found that, if the scale of the mixing is large (macroscopic), then the mixing occurs in a linear fashion. Endmembers consist of those spectra that can be combined in a linear mixing fashion to explain every spectral signature in the data. For microscopic or intimate mixtures, however, the mixing is generally nonlinear [15], [16]. Our conceptual model is that linear mixing occurs inside the observing sensor as photons from disparate materials are co-added (a linear process) to make a single spectrum for a mixed pixel of finite area. Conversely, we see nonlinear mixing happening in the materials themselves as photon path lengths exceed the spatial mixing scales of the materials, resulting in successive absorptions (a nonlinear process). Linear mixing is an observation-induced artifact, and nonlinear mixing is present in the upwelling radiance field whether we measure it or not. Spectral mixture analysis of imaging spectrometer data is in widespread use, with many recent publications describing a 
TABLE I

IMAGING SPECTROMETER Mixing FACtorS

1. An imaging spectrometer integrates the reflected light from each pixel.

2. A very thin volume of surface material interacts with the incident sunlight.

3. All the materials present in this volume contribute to the total reflected signal.

4. Spatial mixing of materials within the GIFOV of the pixel results in spectrally mixed reflected signals.

5. Variable illumination due to topography, shade, and actual shadow in the GIFOV further modify the reflected signal, basically mixing with a variably dark endmember.

wide variety of approaches and applications [17]-[24]. For the purposes of this paper, we are assuming linear mixing (Fig. 1).

A variety of factors interact to produce the mixed signal received by the imaging spectrometer (Table I).

Spectral unmixing consists of determining the mixing endmembers and then estimating their abundances [19]. Unmixing using "known" endmembers is the most widely used method of spectral unmixing [11], [25]. Unfortunately, it typically also requires the most human interaction. To unmix hyperspectral data, the apparent fractional abundance of each endmember material in each pixel is determined, given a set of "known" or assumed spectral endmembers. These known endmembers can be drawn from the data (averages of regions picked by the analyst using previous knowledge) or drawn from a library of pure or mixed materials by interactively browsing through the imaging spectrometer data to determine what materials exist in the image. This method, however, typically requires the knowledge and intervention of an imaging spectrometer "expert." Selection of appropriate endmembers is the most critical and most difficult aspect of successful spectral unmixing [19]. In order to determine the abundances of specific endmember materials, we must first determine what materials are mixing together to give us the spectral signature measured by the instrument. The ideal spectral library used for unmixing consists of endmembers that, when linearly combined, can form all other observed spectra. This can be presented as a simple mathematical model in which the observed spectrum (a vector) is the result of a multiplication of the mixing library of pure endmember spectra (a matrix) by the endmember abundances (a vector; Fig. 2).

An inverse of the original spectral library matrix is multiplied together with the transposes of the orthogonal submatrices and the reciprocal values of the diagonal singular value matrix [25]. A simple vector-matrix multiplication between the inverse library matrix and an observed mixed spectrum gives an estimate of the abundance of the library endmembers for the unknown spectrum. The requirement for significant human intervention can be overcome by taking an iterative approach, followed by statistical analysis of rms error. Kruse et al. [26] describe the iterative use of unconstrained unmixing to arrive at a constrained solution.

To overcome some of the drawbacks inherent in manual selection of endmembers, we have developed an analysis approach for spectral mixing that uses a geometric model and the imaging spectrometer data themselves to "derive" the mixing

\section{Linear Algebra Model of Spectral Mixing}

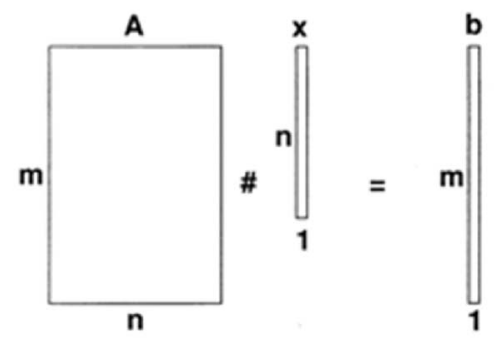

A\# $x=b$

\section{A ( $m$ by $n)$ columns are the endmember spectra \\ $\mathbf{x}(\mathbf{n}$ by 1$)$ is a vector of unknown abundances \\ $b$ ( $m$ by 1 ) is the observed spectrum \\ $m$ is the number of bands \\ $\mathbf{n}$ is the number of endmembers \\ all $x$ values are positive and sum to unity}

Fig. 2. Linear algebra spectral mixing model illustrated graphically and showing the corresponding mixing equation.

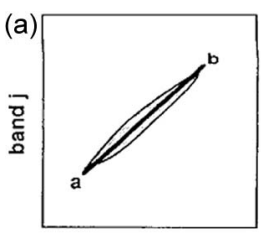

band $\mathrm{i}$

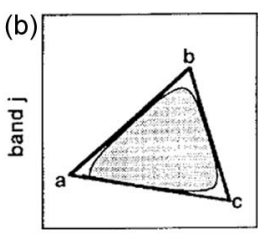

band $\mathrm{i}$

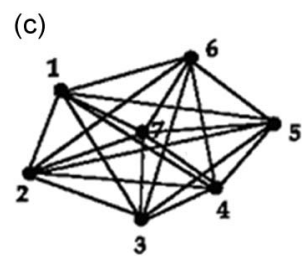

Fig. 3. Geometric model of linear spectral mixing.

endmembers [27], [28]). This methodology is useful as an intuitive and computational tool for finding spectral endmembers in hyperspectral data. Mixed pixels are visualized as points in $n$-dimensional scatterplot space (spectral space), where $n$ is the number of bands. If only two endmembers mix, then the mixed pixels will fall in a line [Fig. 3(a)]. The pure endmembers will fall at the two ends of the mixing line. If three endmembers mix, then the mixed pixels will fall inside a triangle [Fig. 3(b)]. In the case of three or more spectral channels, scatterplots of pairs of channels or linear combinations thereof are merely projections or shadows of the actual higher dimensional data cloud [Fig. 3(c)]. $N$-dimensional spectral analysis seeks to determine the inherent dimensionality of a data set and to analyze it in its native dimensionality. It is the shape of the $n-d$ scatterplot, the patterns within it, and the configuration of its exterior that can be used to understand and analyze the spectral information in the data. The geometric model is extensible to higher dimensions where the number of mixing endmembers is one more than the inherent dimensionality of the mixed data [Fig. 3(c)]. Mixtures of endmembers "fill in" between the endmembers. All mixed spectra are "interior" to the pure endmembers (inside the simplex formed by the endmember vertices) because all of the abundances are positive and sum up to unity. This "convex set" of mixed pixels can be used to automatically determine how many endmembers are present and to estimate their spectra. 
TABLE II

Procedures For THE DERIVED ENDMEMBER APPROACH

1. Correction of imaging spectrometer radiance data to Apparent Reflectance using an atmospheric model, empirical corrections, or other atmospheric corrections

2. Minimum Noise Fraction (MNF) Transform (Spectral compression, noise suppression, and dimensionality reduction)

3. Pixel Purity Index (PPI), Determination of extreme pixels using convex geometric methods (limited iterations for identification of bad pixels)

4. Masking of bad pixels

5. PPI, Determination of "pure" pixels (endmembers( using convex geometric methods (maximum iterations for endmember determination, spatial compression)

6. n-Dimensional Visualization (Endmember Definition) - the automated case involves automatically determining the vertices of the simplex.

7. Identification of endmember spectra using visual inspection, automated identification, and spectral library comparisons

8. Production of material maps using a variety of mapping methods. For the purposes of this description, Mixture-Tuned-Matched Filtering (MTMF) is used for quantification and spatial mapping. MTMF is a partial linear spectral unmixing procedure that finds and maps specific minerals constrained by mixing with a composite (unknown) background. This approach will be described in detail in subsequent sections.

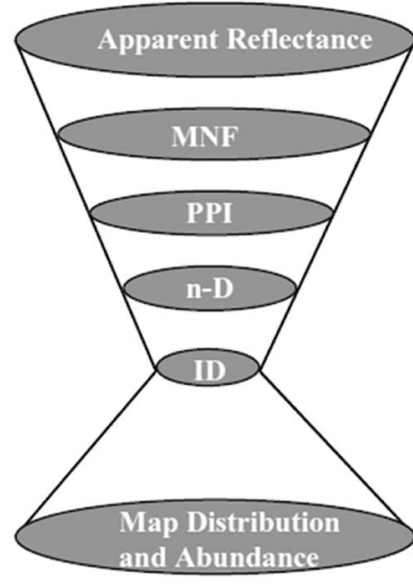

Model-based
Methods
Spectral Data
Reduction
Spatial Data
Reduction
Visualization
Identification
Mapping
Binary, SAM
Unmix
MF \& MTMF
SFF

Fig. 4. $N$-dimensional processing methods for imaging spectrometer data analysis (the "spectral hourglass" approach).

\section{Overview of The ANAlysis ApProACH-The "SPECTRAL Hourglass"}

\section{A. General}

We have successfully implemented the use of convex geometry in an interactive analysis scenario using the derived endmembers (the "spectral hourglass" [3]). This approach starts with the well-conditioned calibrated imaging spectrometer radiance data, converts the data to reflectance, reduces both its spectral and spatial dimensions to just a few key endmembers for identification, and then uses partial spectral unmixing to map the abundance and spatial distribution of the specific endmembers. The steps of this scenario are outlined in Table II and are shown in Fig. 4.

The hourglass method described previously is not the only way to analyze hyperspectral data, but we have found that it provides a consistent way to extract spectral information from hyperspectral data without a priori knowledge or requiring ground observations. The following sections describe the approach in further detail.

\section{B. Atmospheric Correction}

Atmospheric correction is a requirement for most imaging spectrometer analysis applications where the identification of specific materials is desired. Early efforts at removal of atmospheric effects were empirical in nature, limited by the availability of adequate atmospheric models and correction software. Approaches such as the "flat field correction" and "empirical line correction" utilized information from the imaging spectrometer data themselves and field spectra, respectively, to estimate the atmosphere [29]. More recently, well-calibrated imaging spectrometer data and the availability of atmosphericmodel-based approaches have produced excellent reflectance data without specifically requiring the use of ground spectral measurements [30]. Imaging spectrometers typically measure the atmosphere with sufficient resolution to estimate selected atmospheric parameters (water vapor, oxygen pressure altitude, and atmospheric scattering). Used with an atmospheric model, these estimates lead to a per-pixel reflectance corrected data set. While radiance data will also work in spectral mixture analysis, correction to reflectance allows identification of specific materials by comparison to spectral libraries.

\section{Spectral Data Reduction: The MNF Transformation}

The inherent dimensionality of the data is determined using a special orthogonalization procedure related to principal components (the minimum noise fraction (MNF) transform [31]). The MNF transformation is used to determine the inherent dimensionality of the data, to segregate noise in the data, and to reduce the computational requirements for subsequent processing [27], [28], [31]. The MNF transformation can be used to partition the data space into two parts: the one associated with large eigenvalues and coherent eigenimages, and the second with nearunity eigenvalues and noise-dominated images. The noise in the resulting data set has a Gaussian distribution and unit variance. Eigenvalues resulting from the MNF transform describe the intrinsic dimensionality of the data set, i.e., the number of spectral endmembers that account for the majority of the spectral variability in the scene. By using only the coherent portions of the MNF (the highest signal portions) in subsequent processing, most of the noise is separated from the data, thus minimizing the influence of noise on data processing and analysis.

\section{Spatial Data Reduction: Finding Extreme or "Pure" Pixels}

After the MNF transform, the significant bands of the transformed data are subjected to a "pixel purity index" (PPI) investigation, designed to locate the most spectrally extreme (unique or different or "pure") pixels [28]. Based on the aforementioned MNF results, the lower order MNF bands are discarded, and the most significant MNF bands are selected for further processing. These are used in PPI processing. The most spectrally pure pixels typically correspond to mixing endmembers. The PPI is computed by repeatedly projecting $n$-dimensional scatterplots onto a random unit vector. The extreme pixels in each projection are recorded, and the total number of times each pixel is 
marked as extreme is noted. A PPI image is created in which the digital number of each pixel corresponds to the number of times that pixel was recorded as extreme. A histogram of these images shows the distribution of "hits" by the PPI. A threshold is interactively selected using the histogram and is used to select only the purest pixels in order to keep the number of pixels to be analyzed to a minimum. These pixels are used as input to an interactive visualization procedure for separation of specific endmembers. This approach has been implemented as the PPI in the "Environment for Visualizing Images" (ENVI) software, which is a commercial product offered by ITT Visual Solutions, Boulder, Colorado (http://www.ittvis.com) [32].

\section{E. Interactive Visualization: n-Dimensional Scatterplotting}

An interactive " $n$-dimensional" visualization technique is used to extract the endmember spectra using the spectral mixing space. Spectra can be thought of as points in an $n$-dimensional scatterplot, where $n$ is the number of bands [28], [33]. The coordinates of the points in $n$-space consist of " $n$ " values that are simply the spectral reflectance values in each band for a given pixel. The distribution of these points in $n$-space can be used to estimate the number of spectral endmembers and their pure spectral signatures. This geometric model provides an intuitive means to understand the spectral characteristics of materials [Fig. 3(c)].

Operationally, a 2-D projection of the $n$-dimensional scatterplot is interactively rotated in real time to delineate protrusions from the data cloud. The points of these finger-like projections correspond to simplex vertices (thus spectral endmembers). This methodology has been implemented as the " $n$-dimensional visualizer" in ENVI.

\section{F. Identification of Endmembers}

There are many methods for extracting key endmember spectra from hyperspectral data; however, automated identification of these spectra is still problematic. Techniques for direct identification of materials via extraction of spectral features from field and laboratory reflectance spectra have been in use for many years [34]-[37]. They have also successfully been applied to imaging spectrometer data [38]-[43]. We generally use a combination of visual inspection and comparison to spectral libraries, expert knowledge of mineral spectroscopy, and automated methods based on spectral feature matching [43].

\section{G. Conceptual Basis for MTMF}

Background-MF: Matched filtering (MF) was originally developed for target signal detection in signal processing [44][46]. It has recently been extended to hyperspectral exploitation. MF is identical to the constrained energy minimization (CEM) method described by Harsanyi and Chang [47]. It is often referred to as the "optimal" linear detection method for locating a known signature in the presence of a mixed and unknown background. The proofs of such optimality, however, are not applicable to the hyperspectral mixed-pixel problem. They come from the field of electrical engineering and relate directly to the radio and radar matched filter. In these cases,

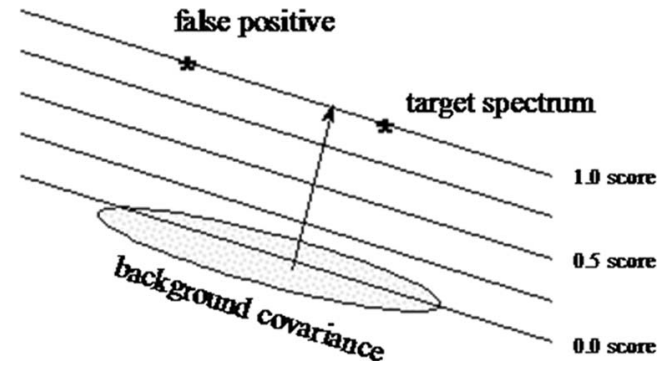

Fig. 5. Diagram illustrating classical matched filter.

the signal is unbounded unlike our mixed pixel that is clearly bounded by the feasibility constraints of unit-sum nonnegative abundance fractions. Thus, while we sometimes use the MF for abundance estimation, we have developed other methods (mixture-tuned MF (MTMF) and other algorithms discussed here) that properly account for mixing.

The standard MF involves calculation of a linear operator or a projection vector, which seeks to optimally balance two objectives: target detection and background suppression. The desired MF output image, which is a linear combination of the input image bands, has several defining properties: zero mean, minimum variance, and perfect target match pixels scored to unity. The standard MF technique uses the covariance statistics of the scene to model the spectral variability of the background. A known target spectrum is projected onto the generalized inverse of the background covariance matrix to derive the MF projection vector (Fig. 5).

This projection of the target spectrum onto the inverse of the background covariance matrix achieves the optimal linear detection vector for the specific target material in the presence of that particular background. The portion of the target spectrum that does not mimic background variations is heavily weighted, while that portion of the target signature that is indistinguishable from the background is strongly suppressed in the resulting filter vector. The MF result, formed by projecting the full data cube onto the filter vector, has the dual properties of a matched filter result: it has minimum variance but also a strong response for pixels that match the target spectrum. The pixels that are dominated by background materials are given near-zero responses, and thus, the signal-to-clutter ratio between the target pixels and the background is maximized. However, other pixels that contain other rare target materials (similar or dissimilar to the target of interest) can often give false positive responses, indistinguishable from actual target responses (Fig. 5). Because these other materials occur in only a few pixels, they do not contribute significantly to the background covariance calculation; thus, they are not nulled properly by the MF method.

A geometric interpretation of the MF method, as shown in Fig. 5, displays the cause of its lack of selectivity. The MF method determines, as described previously, the optimal linear projection vector that separates the target of interest from the background, as described by the scene covariance. To calculate the normalized MF score, all of the spectral data are projected onto this vector, changing each hyperspectral spectrum into a single MF score at each pixel. Then, the score of the data mean spectrum is subtracted to give a zero-mean response to 


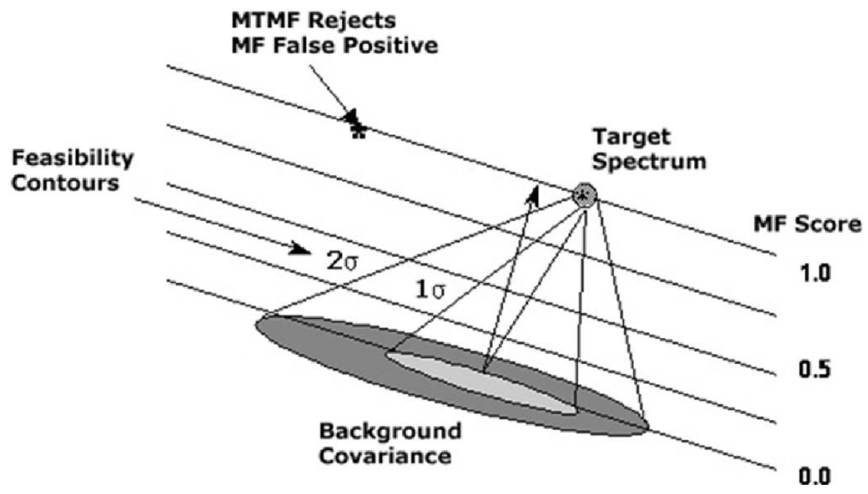

Fig. 6. Diagram illustrating the MTMF concept.

the background. Finally, the data are divided by the score of the target spectrum itself, normalizing the expected scores to range from zero (any background mixture) to unity (a pure target pixel). Since the detection scheme is linear, a half-filled target pixel is expected to score 0.5 in a normalized MF method. The MF vector provides a way of linearly dividing the spectral space into contours (or hypercontours) of equal MF scores. These planes (or hyperplanes) of equal MF scores are all parallel to each other, perpendicular to the MF vector, and parallel to the background covariance. All pixels with spectral responses that fall on the same hyperplane receive the same MF score. This leads directly to the low degree of selectivity among multiple rare targets. Any other rare material (not our target of interest) can have a completely unpredictable score since it is not a covariance driver, and thus, it is not nulled by the MF vector. A spectrum very dissimilar to the target can still get an identical MF score as a perfect target match pixel (a false positive MF score). Discrimination among spectrally similar rare targets is even more difficult for the standard MF method.

MTMF: "MTMF" [48] was designed to leverage mixing between a target of interest and the background in order to resolve the "selectivity" problem inherent in the existing classical MF techniques (also known as CEM and/or orthogonal subspace projection) [46], [47]. The MTMF method recognizes that targets are actually replacing some of the background signature in a pixel (not adding to it as has often been proposed in target detection schemes). This replacement (versus additive) target model is the basis for the MTMF method (Fig. 6). MTMF combines aspects of both hyperdimensional convex geometry spectral unmixing and statistical matched filter target detection to achieve improved performance compared to MF alone. It preserves the ease of calculation of the MF and allows for enhanced target selectivity, excelling at accurate mapping of extremely small subpixel targets with very low false alarm rates. MTMF often produces low false alarm rates when used on the same input data used for other applications that previously suffered from numerous false alarms [48]. This performance improvement is indicative of the power of the mixture tuning (MT) aspect of the approach. To fully characterize the detection versus false alarm rejection, even more challenging applications are required. A general conceptual overview is given here, the theory behind MTMF is described, and a detailed step-by-step explanation of the algorithms that make up MTMF is outlined in the following sections.
As stated previously, MTMF combines the statistical method of the MF with the deterministic method of the linear mixing model. The one endmember that we do know is the known target of interest. We use the background statistics, just as in MF, to define the variability in the unknown natural and dominant endmembers. We use the known signature of the target material to define one corner of a mixing simplex. The background covariance (or equivalently eigenvectors and eigenvalues) gives the shape, orientation, and size of the background spectral scatter. The known target material is a fixed point with a wellknown noise distribution. Since the MTMF works with data that have been whitened and decorrelated with the MNF transform, the noise distribution about a known point is just a unit variance isotropic Gaussian distribution. The MF vector and associated scores are calculated as before. Now, in addition, we calculate an infeasibility number, describing in terms of noise standard deviations how likely it is that each pixel is a mixture of our known target and the background materials. This is done by linearly "morphing" the unit variance noise distribution of the known target into the measured background distribution as the MF scores range from one to zero. Another way to envision the method is to imagine hypercones of equal probabilities converging on the target spectrum and diverging away to meet the background distribution (Fig. 6).

To get the infeasibility score for a certain pixel, first, its MF is found, and then, its distance from the line that connects the mean of the background and the target is measured. This measurement is done in the plane of the fixed MF score and is done in terms of number of noise standard deviations of the linearly morphed distribution for that MF score. A pixel outside the feasibility hypercone, although it does still get a perfect MF score, is shown to be false positive since it is highly infeasible as a mixture of the target spectrum and the background distribution (Fig. 6). The MTMF is very useful for detection and discrimination among multiple rare targets with low spectral contrast between targets and background and among targets. This approach has been implemented as "MTMF" in ENVI.

\section{MTMF THEORY AND AlgORITHMS}

The MTMF method can be divided into three main steps: preprocessing and data conditioning, MF for abundance estimation, and MT for false positive rejection. The goals and objectives of the three steps are outlined here and in Table III, and the implementations of the methods are discussed in detail in the following sections. The first step is conducted via an MNF transform [31]. The second step consists of a matched filter calculation (MF) using the MNF data statistics as the background statistics. The final step is an MT. It is unique to MTMF and uses convex geometry theory to measure the feasibility of each pixel as a mixture of the target and background, revealing false positives and improving subpixel detection.

While the approach has three main steps, each with several substeps, operationally, it is very simple. The user only needs to have the data to be processed and one or more target spectra. The MNF and MTMF processes require no user intervention. The following sections detail each processing step. 
TABLE III

MTMF PROCESSING STEPS

1) Preprocessing and Data Conditioning (MNF Transform) [31]

a) Estimation of noise covariance matrix

b) Data subjected to noise whitening transform

c) Mean subtracted from noise-whitened data

d) Noise-whitened data projected onto its eigenvectors, decorrelating it

2) Matched Filtering for Abundance Estimation (MF Projection) [44]-[47]

a) Target spectrum transformed to MNF space

b) Background covariance matrix estimated and inverted

c) Normalized matched filter projection vector calculated

d) MNF data projected onto MF vector to estimate target abundances

3) Mixture Tuning to Measure Feasibility (MT Modeling)

a) Continuum of mixture distributions interpolated

b) Mixture infeasibilities calculated for each pixel

c) Valid detections separated from false positives

\section{A. Preprocessing and Data Conditioning (MNF Transform Implementation) [31]}

The two objectives of this preprocessing are noise whitening and data decorrelation. Noise whitening is accomplished by estimating and applying an affine transform to the original data, which makes the noise in the data uncorrelated, and unit variance in all spectral dimensions. It is critical for MTMF to have the noise in the data well-characterized and whitened. The noise model forms a key element of the MT portion of the process and allows for quantitative estimation of detection probability and spectral contrast measures.

The first step in the MNF transform is the estimation of the noise covariance matrix and its decomposition into its eigenvalue vector and eigenvector matrix. There are three possible methods for this process: 1) the estimation of noise statistics from the data via a shift difference; 2) the estimation of noise statistics from a dark current image; or 3) the application of known noise statistics. The first method (shift difference) can be used if no appropriate auxiliary data are available, estimating the noise statistics from the data themselves. The second method uses a dark current image to characterize the noise in the data. The third method is useful for simulations or cases where the noise statistics are known a priori.

The shift difference uses the mean of a row shift difference and a column shift difference to estimate the noise in the data. The input to the method is the data set itself $\mathbf{D}$ (a 3-D matrix of spectral data which is ns, nl, and nb, where ns, nl, and nb are the number of samples, lines, and bands used). The method can be understood to create a "noise-only" data set $\mathbf{N}$ whose dimensions are $\mathrm{ns}-1, \mathrm{nl}-1$, and $\mathrm{nb}$, allowing for the extra column and row associated with the shifting. Each band of $\mathbf{N}$ is the average of the row shift difference and the column shift difference of the same band of $\mathbf{D}$

$\mathbf{N}_{i, j, k}=\left(\left(\mathbf{D}_{i, j, k}-\mathbf{D}_{i-1, j, k}\right)+\left(\mathbf{D}_{i, j, k}-\mathbf{D}_{i, j-1, k}\right)\right) / 2$

where

$i \quad$ sample number and ranges from two to ns;

$j$ line number and ranges from two to nl;

$k$ band number and ranges from one to $\mathrm{nb}$ or equivalently

$$
\mathbf{N}_{i, j, k}=\mathbf{D}_{i, j, k}-0.5 * \mathbf{D}_{i-1, j, k}-0.5 * \mathbf{D}_{i, j-1, k} .
$$

The method implicitly assumes that the signal portions of the data from neighboring samples or lines are identical and thus cancel in the subtraction, but the noise is not identical and remains in the shift difference result. Of course, some "leakages" of the signal into the noise estimate does occur, but this problem is usually not too severe. Using an image subset that is relatively homogeneous, with a minimum of high spatial frequency content, can minimize signal leakage. The desired covariance noise matrix $\mathbf{S N}$ is calculated from $\mathbf{N}$ in the standard manner, treating $\mathbf{N}$ as a 2-D matrix of np by nb, where $\mathrm{np}$ is the total number of pixels in the shift difference result $[(\mathrm{ns}-1)$ times $(\mathrm{nl}-1)]$. The elements of the shift difference noise covariance matrix must be divided by 1.5 to account for the squaring of the three terms in (2) $\left(1.5=1^{2}+0.5^{2}+\right.$ $\left.0.5^{2}\right)$. Noise covariance matrices estimated from dark images or experimental sources would not need this scaling

$$
\mathbf{S N}_{i, j}=\left(\Sigma\left(\mathbf{N}_{k i}-n_{i}\right) *\left(\mathbf{N}_{k j}-n_{j}\right) /(n p-1)\right) / 1.5
$$

where

$i$ and $j$ range from one to $\mathrm{nb}$;

$k \quad$ ranges from one to np;

ni mean value of band $i$ of the noise image $\mathbf{N}$.

The alternative methods of estimation of SN require the user to supply auxiliary information. If the user chooses to employ a dark current image, it is used as a direct proxy of $\mathbf{N}$. Of course, this image must be scaled to match the units of the data, including any scale factors applied to convert raw DN to radiance and radiance to scaled reflectance. The third alternative allows the user to directly provide an estimate of the noise covariance matrix $\mathbf{S N}$.

The next step in data preprocessing is the calculation of the eigenvalues and eigenvectors of the estimated noise covariance matrix SN. We use a two-step process for eigenanalysis of positive, definite, and symmetric matrices such as covariance matrices, as suggested in the standard text Numerical Recipes [49]. The two steps are the reduction of the covariance matrix to tridiagonal form via a series of householder transforms, followed by a diagonalization using QL transforms with implicit shifts. We also sort the eigenvalues and associated eigenvectors, reporting them in order of decreasing eigenvalue. The results of this decomposition are the noise eigenvalue vector Neval and the noise eigenvector matrix Nevec.

The original data are next mean corrected and noise whitened. This is achieved by subtracting the average spectrum $\mathrm{dm}$ from each data spectrum. Then, the mean-corrected data are projected onto the noise eigenvector matrix Nevec, decorrelating the noise in the data. Finally, each resulting band is divided by the square root of the associated noise eigenvalue, normalizing the noise variance to unity in each band. These processes produce zero-mean noise-whitened data, as shown in the following equation:

$$
\operatorname{Dmcnw}_{i, j}=\mathbf{N W} * \mathbf{N e v e c} *\left(\mathbf{D}_{i, j}-\mathbf{d m}_{j}\right)^{\mathrm{T}}
$$


where

$\mathbf{D}_{i, j} \quad$ original data as np, nb matrix;

$\mathrm{np} \quad$ total number of pixels $(\mathrm{ns} * \mathrm{nl})$;

$\mathbf{d m}_{j} \quad$ mean value of band $j$ of $\mathbf{D}_{i j}$;

Nevec nb, nb matrix of noise eigenvectors;

NW diagonal matrix, where its elements are the square root of the reciprocals of the noise eigenvalues;

Dmcnw mean-corrected noise-whitened result.

The final step in the MNF preprocessing is a standard principal component transform of the mean-corrected noisewhitened data Dmenw. This is conducted in the standard way, calculating the covariance matrix of Dmcnw and using the methods described previously to retrieve the associated eigenvectors and eigenvalues (Dmcnweval and Dmcnwevec). Finally, the mean-corrected noise-whitened data are projected onto their eigenvectors, decorrelating the data and providing the final MNF output

$$
\text { Dmnf }=\text { Dmcnwevec } * \text { Dmcnw. }
$$

The MNF data Dmnf have several unique and useful properties. First, they contain uncorrelated unit variance white noise. Second, they have a zero mean. Third, they are uncorrelated, being projected onto their own eigenvectors. Thus, they now have a diagonal covariance structure, with covariance values equal to the MNF eigenvalues Dmcnweval. The MNF data are the basis for all following MTMF processing. In fact, MTMF processing cannot proceed without data that exhibit these unique properties resulting from the preprocessing. Noise whitening is especially useful since it provides a simple noise model (isotropic unit variance that is used in subsequent mixture feasibility modeling). The zero-mean and diagonalcovariance properties are also used to speed up and to simplify the subsequent MTMF process.

Although it is made up of several steps and their associated transformations, the MNF transform can be viewed as a single, composite, and affine transform of the original data to its MNF representation. This single transformation can be modeled as a subtraction of the mean spectrum $\mathbf{d m}$ (mean correcting the data), and a matrix multiplication by a composite operator Tmnf that combines the noise whitening rotation and scaling and the final MNF decorrelation

$$
\operatorname{Dmnf}_{i, j}=\operatorname{Tmnf} *\left(\mathbf{D}_{i, j}-\mathbf{d m}_{j}\right)
$$

where

Tmnf composite MNF scaling and rotation matrix;

$\mathbf{D}_{i, j} \quad$ original data;

dm mean spectrum of $\mathbf{D}$.

\section{B. MF for Abundance Estimation (MF Projection)}

The MTMF process can best be understood as two separate steps: MF for abundance estimation (MF) and MT for false positive rejection (MT). The first step (MF) is described in detail here. MT is described in the following section. As previously discussed, MF is often referred to as the "optimal" linear detection method for locating a known signature in the presence of a mixed and unknown background. This description, however, comes from the field of electrical engineering and relates directly to the radio and radar matched filter. In these cases, the signal is unbounded. For the optical mixed-pixel case, however, the MF must be bounded by the feasibility constraints of unitsum nonnegative abundance fractions. While we use the MF for abundance estimation, the real power of the MTMF method is the MT that exploits this extra information. MTMF uses MF for abundance estimation and background suppression and MT for false positive identification and rejection.

The standard MF involves calculation of a linear operator or projection vector that seeks to optimally balance two objectives: target detection and background suppression. The desired MF output image, which is a linear combination of the input image bands, has several defining properties: zero mean, minimum variance, and perfect target match pixels scored to unity. Solving for these constraints leads to the standard MF projection vector equation

$$
\mathbf{M F V}=\left(\mathbf{C}^{-1} *(\mathbf{t s}-\mathbf{d m})\right) /\left((\mathbf{t s}-\mathbf{d m})^{\mathrm{T}} * C^{-1} \#(\mathbf{t s}-\mathbf{d m})\right)
$$

where

MFV desired MF projection vector;

$\mathbf{C}^{-1} \quad$ generalized inverse of the data covariance matrix;

ts target spectrum;

dm mean spectrum of the data.

This result gives us the single projection vector that exhibits the desired properties. When the full data scene is mean corrected and projected onto this vector, it produces the MF abundance image. As desired, this image will have a zero mean and an optimal, in a least-squares sense, balance between image variance and target to background contrast. The process of projecting the mean-corrected target spectrum onto the generalized inverse of the data covariance achieves this balance by finding that portion of this contrast vector that is perpendicular to the space spanned by the data covariance. This projection optimally balances target-background separation and output image variance. Geometrically, it returns a projection vector that weights the dimensional components of the contrast vector inversely with the eigenvalues of the data. The denominator of (7) is the raw MF score of the target spectrum. Normalizing the MFV by this scalar assures that the final MFV will give unit scores for pixels that are perfect matches for the target. As will be shown later, the MF unfortunately also gives perfect scores to an infinite set of other possible spectra. It is this ambiguity of the MF that opens the door for false positives and permits the dramatic improvement that the MT contributes to the MTMF method.

Since MTMF applies MF to MNF data, the classical MF method described previously can be simplified and accelerated. The MNF data are already zero mean and uncorrelated. Thus, the covariance and mean of the MNF data are known, and they have a simple and special form. The covariance matrix of the MNF data Cmnf is simply a diagonal matrix, whose elements are the MNF eigenvalues Dmcnweval. Since the noise in the MNF data is whitened and unit variance, the diagonal elements of $\mathrm{Cmnf}$ are all greater than or equal to one, with no near-zero values. Thus, the generalized inverse of $\mathbf{C m n f}\left(\mathbf{C m n f}^{-1}\right)$ is again a diagonal matrix, with elements equal to the reciprocals 
of the eigenvalues Dmcnweval. The MF projection vector is then calculated by transforming the target spectrum into the MNF space using the affine transform shown in (6), then by projecting it onto the inverse of Cmnf, and again by normalizing it by the raw score of the converted target spectrum

$$
\mathbf{M F V}=\left(\mathbf{C m n f}^{-1} * \mathbf{t s m n f}\right) /\left(\mathbf{t s m n f}^{\mathrm{T}} * \mathbf{C m n f}^{-1} * \mathbf{t s m n f}\right)
$$

\section{where}

MFV desired MF projection vector;

Cmnf $^{-1}$ diagonal inverse of the MNF data covariance matrix;

tsmnf target spectrum converted to the MNF space $(\mathbf{t s m n f}=\mathbf{T m n f} *(\mathbf{t s}-\mathbf{d m}))$

ts original target spectrum;

dm mean spectrum of the data.

Using MNF data for MF processing has two distinct advantages. The noise whitening preconditions the data, so inversion of the data covariance matrix is straightforward and stable unlike the inversion of a typical covariance matrix of raw hyperspectral data. Second, the scale of the units of the MNF data permits direct and quantitative determination of detection probabilities and performance metrics because the amplitude of one DN in each MNF band is identical to the standard deviation of whitened and rescaled noise.

The abundance values for the target detection in MTMF are now determined by projecting the MNF data onto the MF projection vector described in (8). This leads to the first half of the final MTMF two-part result [the MF target apparent abundance image (MF)]. The values of this image have a zero-mean minimum variance distribution. Pixels that are perfect target matches will score one, and pixels that are half-filled will score 0.5

$$
\text { MFI }=\text { MFV } * \text { Dmnf }
$$

where

MFV matched filter vector;

Dmnf MNF data set.

This MF result image MFI forms the basis for our target abundance determination. Numerous false positives, however, are common in such results. Since the MF is a linear filter, the entire hyperplanes of pixels map to single MF answers. The MF vector simply contours the spectral space, scoring each pixel according to its distance from the background, in the special direction specified by MFV. MF false positives are common, especially in the cases of spectrally similar targets for subpixel abundances. Tuning the results using a geometric mixing model greatly reduces the false positive problem inherent in MF processing, giving the outstanding results demonstrated by the MTMF.

\section{MT to Measure Feasibility (MT Modeling)}

The most powerful and unique aspect of the MTMF methods is its MT, leveraging a high-dimensional convex geometry model of spectral mixing. MT permits the direct identification and rejection of the false positives that are common in MF results. This extra information, beyond the allegedly "optimal" results of the MF, is achieved by exploiting the underlying physics of the hyperspectral mixed-pixel problem. Unlike the wholly unbounded radar and radio signal problem, mixed pixels have quite different underlying controls on their spectral distributions. The observable radiance, the surface reflectance, and even the MNF representation of the data are merely affine transformations of the underlying variables of interest (the abundances of each material in each pixel). These abundances must obey two simple, yet powerful, constraints. First, the abundances must be nonnegative. Second, the abundances for each pixel must sum up to one. These two constraints (nonnegativity and unit-sum) are the feasibility constraints.

The feasibility constraints imply that the spectral scatterplots of mixed-pixel data must occupy $n$-dimensional simplexes when $n+1$ endmembers are present in a scene. Two endmembers lead to a 1-D line segment mixing simplex. Three endmembers give rise to a 2-D triangular mixing simplex. Four endmembers create a tetrahedral 3-D mixing simplex, etc. In higher dimensions, the same concept carries forward: each endmember adding a vertex to the convex mixing simplex and an associated dimension. This fact is a direct consequence of the feasibility constraints. As a pixel fills with a certain material, its degree of spectral variability is reduced. As a pixel fills to $100 \%$ target material, all mixing freedom is lost, and any variability must be due to noise. If a pixel has $0 \%$ target material, the maximum mixing freedom exists, and all permitted feasible mixtures of the other $n$ endmembers are possible. This $0 \%$ target pixel must then lie in the $n$-dimensional simplex that forms the facet opposite the target endmember vertex. Similarly, a pixel that has $50 \%$ target material will have mixing freedom that is exactly halfway between the noise-only freedom of the pure target pixel and the background-plus-noise freedom of the $0 \%$ pixel. MTMF exploits this concept, but it does not require knowledge of the specific spectra of the background endmembers. Pixels that fall far outside the range of their allowed mixing freedom can be identified and recognized as false positives. They appear as infeasible mixtures of the target and background. The feasibility constraints and the related convexity concepts form the foundation of the MTMF MT.

MTMF creates a range of distributions describing in a statistical sense (via a mean spectrum, a matrix of eigenvectors, and a vector of eigenvalues) the expected feasible mixing range for pixels as a function of their MF apparent abundance scores. This range of distributions is bounded at one end by the zeroabundance distribution, described by the background mean, eigenvectors, and eigenvalues. This extreme distribution is used for pixels that score zero or lower in the MF calculation. The other extreme distribution, in the continuous range of distributions used by MTMF, is that for the $100 \%$ abundance pixels. This pure target mixing distribution is noise only and is characterized by the mean target spectrum in MNF space and a set of $n b$ orthogonal unit eigenvectors and a set of unit eigenvalues. Here, we take advantage of the noise whitening of the MNF preprocessing. If a pixel is a pure target pixel, it must be within a few standard deviations (a few MNF DN) of the target spectrum since no mixing freedom is allowed in this pure pixel. The noise-only mixing distribution is used for all pixels scoring one or more in the MF processing. Intermediate MF abundance values have associated with them intermediate 
mixing distributions. These intermediate distributions are created by linear interpolation of the large ellipsoidal $0 \%$ abundance background distribution and the small spherical $100 \%$ abundance noise distribution. The intermediate distributions use the eigenvectors of the background distributions, but they have eigenvalues that are reduced so that the square roots of the eigenvalues range smoothly from their background values to unity as the MF scores change from zero to one.

MTMF performs its MT by calculating a number that directly measures the mixing infeasibility for each pixel. This infeasibility number is simply the distance of that pixel from the line segment connecting the target spectrum and the background mean, measured in terms of standard deviations using the appropriate mixing distribution for the MF score of that pixel. If the pixel scored one or more in the MF process, indicating a potentially pure pixel, the appropriate distribution is the MNF noise distribution. In this case, the MT infeasibility score is simply the Euclidean distance of the pixel from the target mean spectrum, in MNF units, or noise standard deviations. If the pixel scored zero or less in the MF process, indicating a pixel with no target material present, the appropriate distribution is the background distribution, given by the data mean, eigenvectors, and eigenvalues. In this case, the MT infeasibility score is the Mahalanobis distance of the pixel from the data mean. Numerically, this is the number of standard deviations from the mean to this pixel. This is calculated by projecting the pixel onto the eigenvectors, dividing this projected spectrum element by element by a vector whose elements are the square roots of the data eigenvalues. Then, the MF infeasibility score is taken as the square root of the sum of the squares of this scaled vector. Again, however, this is simply the number of standard deviations from the mixing line that connects the background mean to the target spectrum. Pixels with intermediate MF scores between zero and one must use interpolated distributions for this infeasibility measurement.

The detailed calculation of the MT infeasibility score involves three steps: determination of the mean of the appropriate distribution, interpolation of the eigenvalues of the appropriate distribution, and measurement of the separation of the pixel from the mean, in terms of standard deviations of the appropriate distribution, via projection onto the scaled eigenvectors. For each pixel, first, the appropriate mean of the distribution is calculated, interpolating linearly between the mean of the background (zero in the case of MNF data) and the target spectrum

$$
\operatorname{adm}_{i}=\operatorname{MFI}_{i} * \mathbf{t s m n f}
$$

where

MFI $_{i} \quad$ MF result for the $i$ th pixel, i.e., a scalar;

tsmnf target spectrum in the MNF space;

$\mathbf{a d m}_{i} \quad$ appropriate distribution mean for pixel $i$ given its MF score.

Note that (10) illustrates the case for MNF data only, assuming a zero-mean background. This describes a parametric equation mapping a range of values for adm, from the zero spectrum to tsmnf as the MF score ranges from zero to unity. In other possible cases, where the background mean is not zero, (10) must be linearly modified to have the resulting adm spectra range from the nonzero background mean to tsmnf as the MF scores range from zero to one.

The second step in the MT calculation is the interpolation of the eigenvalues for the appropriate distribution, given the MF score for the particular pixel. This interpolation is done so that the square roots of the eigenvalues range linearly from the background values sqrt(Dmcnweval) to ones (the MNF noise eigenvalues, all ones) as the MF scores range from zero to one

$$
\begin{aligned}
\operatorname{MTeval}_{i}= & \left(\text { Dmcnweval }^{1 / 2}\right. \\
& \left.-\mathbf{M F I}_{i} *\left(\text { Dmcnweval }^{1 / 2}-\mathbf{U V}\right)\right)^{2}
\end{aligned}
$$

where

Dmcnweval vector of MNF eigenvalues;

MFI $_{i} \quad$ MF score of the $i$ th pixel floored at zero, ceilinged at one;

UV vector of ones (the MNF noise eigenvalues).

We linearly scale the L1-norm eigenvalue square roots to give a mixing hypercone with linear cross section in data space, whereas linearly scaling the L2-norm eigenvalues would give a nonlinear hypercone cross section. The MT builds an approximation of that vertex of the linear mixing simplex associated with the target spectrum using the background mean and covariance as a proxy for the location and orientation of the opposite simplex facet.

The final step in the MF infeasibility calculation involves the subtraction of the appropriate mean from the data value, projection onto the eigenvectors, division element-by-element by the square roots of the eigenvalues, and calculation of the Euclidean length of the result. In our special case of MTMF modeling of the MNF data, this process is simplified since the MNF data are already projected onto their eigenvalues

$$
\mathbf{M T}_{i}=\left\|\left(\mathbf{D m n f}_{i}-\mathbf{a d m}_{i}\right) /\left(\mathbf{M T e v a l}_{i}\right)^{\frac{1}{2}}\right\|
$$

where

Dmnf $_{i} \quad$ MNF spectrum for pixel $i$;

$\mathbf{a d m}_{i} \quad$ appropriate mean for pixel $i$ as described in (10); $\mathbf{M T e v a l}_{i} \quad$ interpolated vector of eigenvalues for pixel $i$.

The final value $\mathbf{M T}_{i}$ is then the number of standard deviations from the target background mixing line for each pixel, measured using appropriate interpolated eigenvalues based on the MF score at that pixel. The MT score can be directly interpreted to indicate mixing feasibility. Low scores, indicating low infeasibility, are associated with feasible mixtures. High MT scores, which mean a separation of many standard deviations from the mixing simplex, are indicative of infeasible mixtures and can be used to identify false positives.

The MT and MF scores can be jointly interpreted to provide high-performance subpixel target detection and extreme false alarm rejection. Each portion of the MTMF result provides independent and different information. The MT result answers the question, "Is this pixel a feasible mixture of the target material and the scene background?" The MF result answers the question, "How much of this material, if it is present, appears to be in the pixel?" Pixels that satisfy the first question, by showing a suitably low MT score, indicating a feasible 

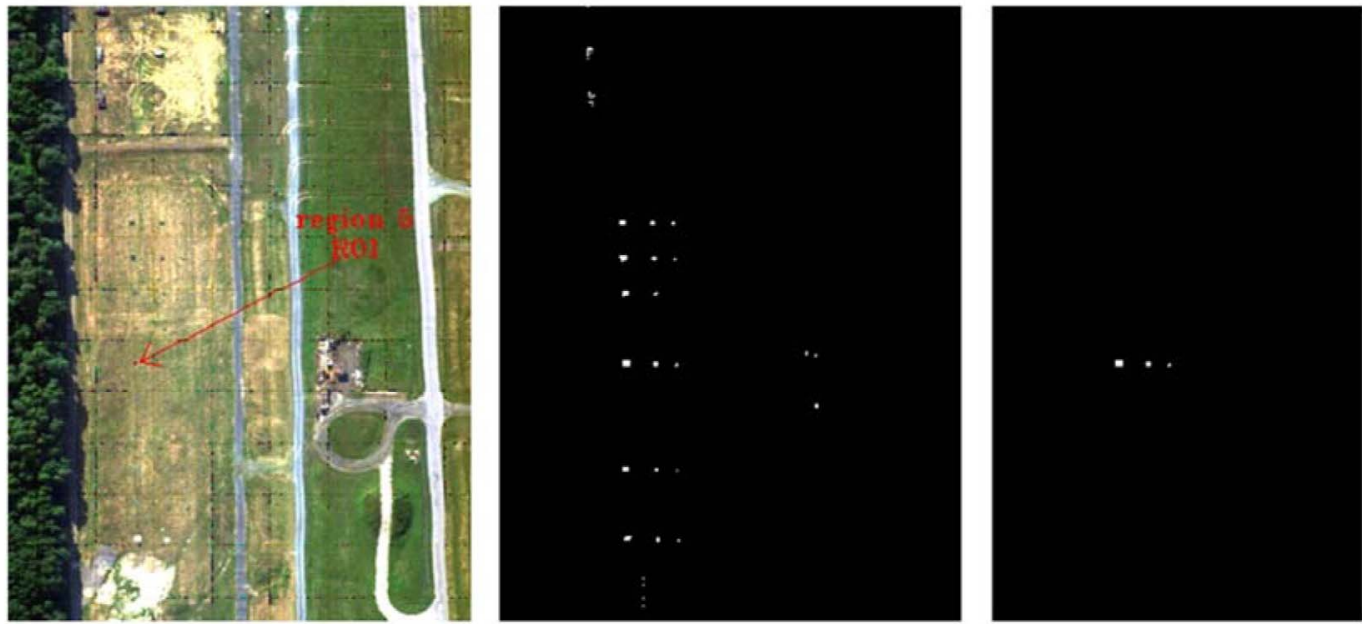

Fig. 7. (a) True color image of a portion of run05, HYDICE Forest Radiance data, showing region 5 defining ROI. The same known materials occur in three progressively smaller areas from left to right. (b) MF result for the region 5 ROI endmember, showing many false alarms at the 0.069 ( $\sim 7 \%$ abundance) MF threshold. Note the three sets of targets and confusion between these. (c) MTMF result combining high MF and low infeasibility. Note the three rows of the same region 5 material mapped using the MTMF approach. There are no false alarms in this case using the $0.069(\sim 7 \%)$ threshold and the mixing feasibility constraint.

mixture, can then give reliable MF abundance estimations. The MT result is especially useful for identifying and rejecting MF false positives and for permitting robust subpixel detection at very low abundance and spectral contrast levels. The MTMF performance will depend on the accuracy of the supplied endmember and on the degree of linearity of the spectral mixing involved.

\section{Application Examples}

\section{A. Case History \#1: Target Detection}

The power of the MTMF method is best demonstrated by its performance on real data. HYperspectral Data Imagery Collection Experiment (HYDICE) data of run05 of the Forest Radiance experiment were analyzed using the MTMF approach. HYDICE was a nadir-viewing 210-channel push broom imaging spectrometer covering a spectral range from 0.4 to $2.5 \mu \mathrm{m}$ [50]. The spectrum is sampled contiguously in approximately 10-nm-wide bands. HYDICE instrument performance characteristics are discussed in detail in [51]. The Forest Radiance I experiment was conducted in August 1995 at the U.S. Army's Aberdeen Proving Grounds in Maryland [50]. HYDICE data were collected in a controlled environment in a woodland forest setting with multiple targets of known materials interspersed. The reflectance data provided by the HYDICE project were calculated using an empirical correction to known ground targets. Fig. 7(a) shows a reference image of a portion of the scene. In this case, image endmember spectra were interactively defined by choosing image pixels associated with a known field target as a region of interest (ROI). The data were subjected to an MNF transform using 150 of the bands, dropping the noise-only bands in the water absorption regions. Inspection of the MNF data revealed that MNF band 1 was wholly dominated by instrument noise, including across-track calibration error and down-track "heartbeat." This MNF band was dropped for subsequent processing. MTMF was run on a subset of the MNF bands, calculating the MF and infeasibility

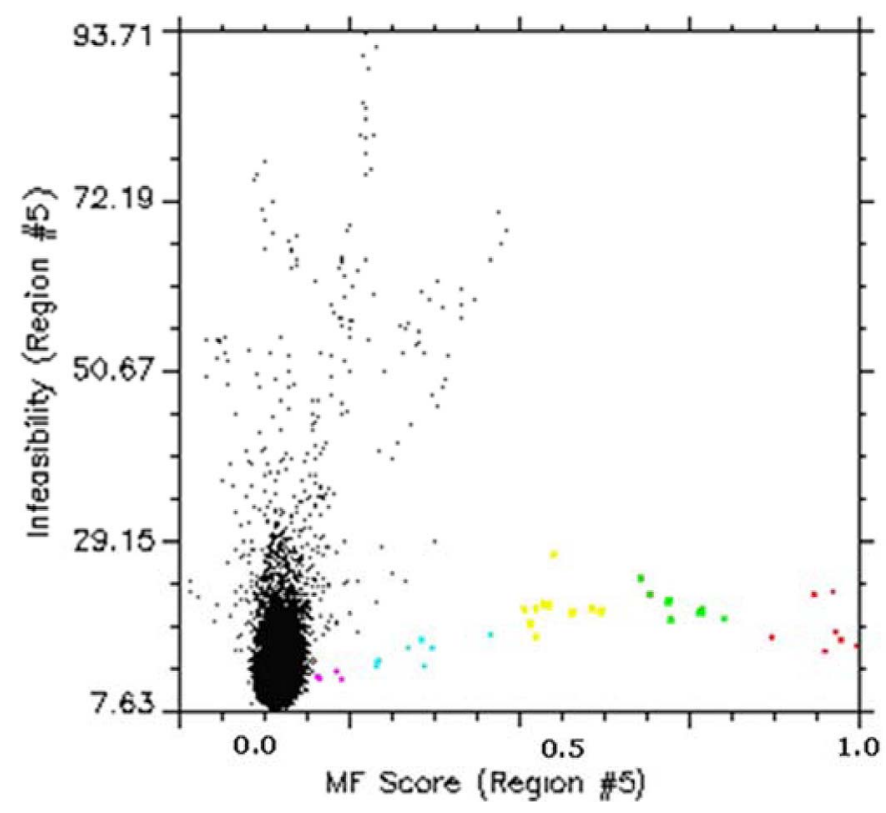

Fig. 8. Scatterplot of MF abundance versus infeasibility scores. The highlighted color pixels represent those materials with low infeasibility and progressively higher MF scores to the right. These are pixels mapped using the MTMF.

scores for each pixel as compared to the known ROI (region 5). There are three progressively smaller targets (left to right) with these known spectral characteristics [Fig. 7(b) and (c)]. The results show the highly selective nature of the MF versus MTMF algorithms. In this case, using the same abundance threshold for both MF and MTMF, no false alarms were seen for the MTMF, which incorporates the mixing feasibility constraint [Fig. 7(b) and (c)].

The MTMF result is also shown in a scatterplot form in Fig. 8. The MF abundance is on the horizontal axis. The MT infeasibility score is on the vertical axis. The valid detections are along the bottom of the plot (MF abundances ranging from zero to one), with consistently low infeasibility scores. 


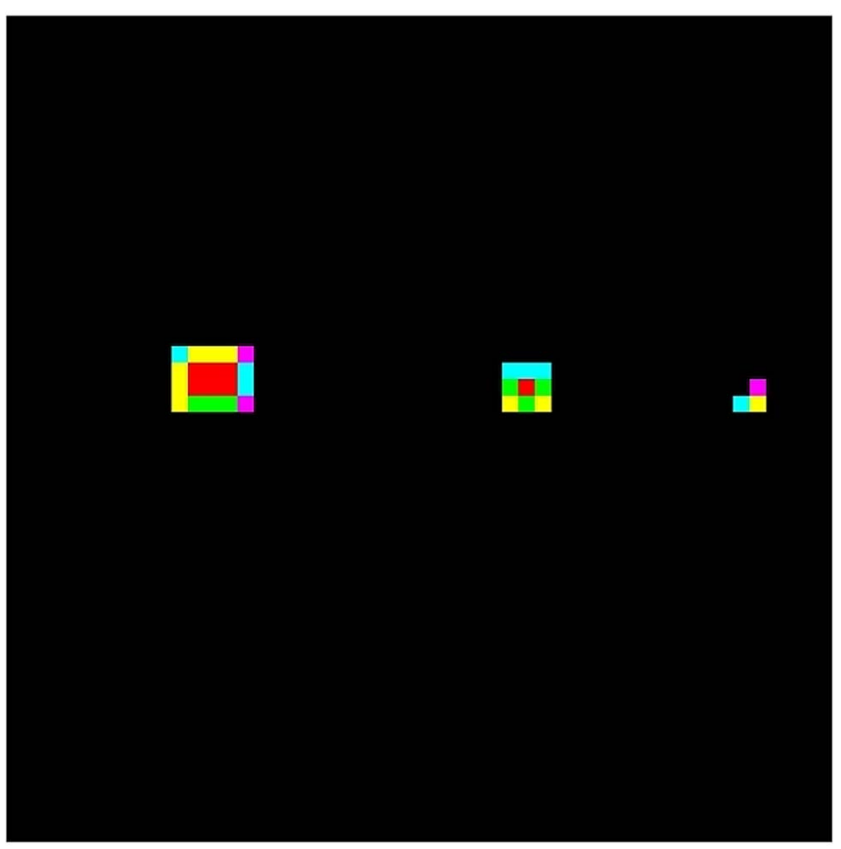

Fig. 9. Close-up view of extended targets from Fig. 7(c), showing robust subpixel detection on edges. Colors correspond to Fig. 8 colored pixels.

Those pixels that would map as MF false positives are those that stream up and to the right, out of the background cluster. These pixels have statistically significant MF scores, but the MT process has revealed them as false positives by their high mixture infeasibilities.

By including the MT portion of the process, the target detection is much improved [Fig. 7(c)]. MTMF effectively reduces the minimum fraction detectable with no false alarms to 0.069 ( $\sim 7 \%$ abundance) from the value of 0.407 ( $\sim 40 \%$ abundance) using only an MF approach. Conversely, if the MF threshold is set to include all 33 pixels correctly found by the MTMF method without false alarms, 140 false positives are also found by using MF alone (Fig. 7). The MTMF method permits detection, in this case, down to abundances of 0.069 with no single false alarm. While MTMF would also begin showing false alarms below this threshold, the higher false alarm rate using only the MF method emphasizes the key role of insuring feasible mixtures using the MTMF in reducing false alarms. Fig. 7(a) and (b) shows a comparison of the MF and MTMF results, each using the same cutoff threshold.

The MTMF method is so robust that it can even be used to give subpixel locations of extended multipixel targets. Fig. 9 shows the three targets (of the same material) detected using the MTMF approach. Color density slicing the MF versus MT infeasibility scatterplot (Fig. 8) produces the color-coded image (Fig. 9), which shows that there are only a few pixels at the center of the two larger targets that have both high MF and low infeasibility scores, indicating a complete pixel fill (pure pixels). All of the surrounding pixels (still part of the known target) have lower abundances because of pixel mixing. The colors in the scatterplot match those in the image display, with red pixels indicating abundances greater than about $90 \%$, green showing abundances between approximately $60 \%$ and

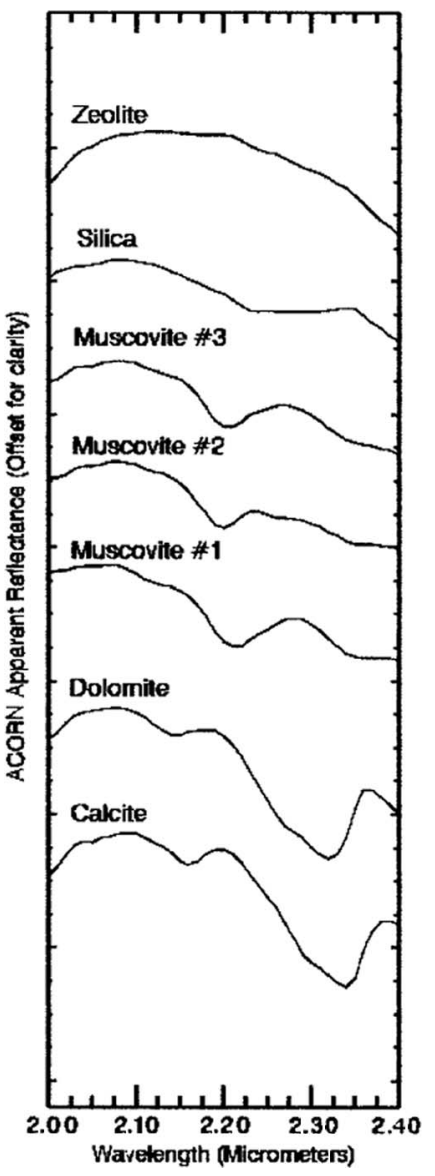

Fig. 10. Mineral endmembers extracted for the NDV site using the spectral hourglass approach.

$90 \%$, yellow equaling abundances ranging from about $40 \%$ to $60 \%$, cyan showing abundances between approximately $20 \%$ to $40 \%$, and magenta from about $10 \%$ to $20 \%$ abundance.

\section{B. Case History \#2: Mineral Mapping, NDV, NV/CA}

MTMF can also be used for mapping natural targets, with high confidence in detection and mapping of specific materials. A site in northern Death Valley (NDV) is used to illustrate the basic results of mineral mapping using MTMF. This area is approximately halfway between Reno and Las Vegas, NV, directly on the CA-NV border, within the Death Valley National Park. It has previously been observed by numerous remote sensing instruments, and significant ground truth exists [3], [38], [52]. Precambrian bedrock in the area consists of limestones, dolomites, and sandstones. A Mesozoic quartz monzonite-composition stock was intruded into these at depth, altered by hydrothermal fluids (quartz-sericitepyrite or "QSP" alteration), and then eroded to its present surface exposures [38]. Minor copper mineralization occurs along NW-trending fractures. The AVIRIS data collected on June 9, 2000, at 20-m spatial resolution were analyzed using the image-based spectral endmember and convex geometry approach described previously. AVIRIS is a whisk broom imaging spectrometer covering the $0.4-2.5-\mu \mathrm{m}$ range with $10-\mathrm{nm}$ spectral resolution and 2-20-m spatial resolution [53]. The 

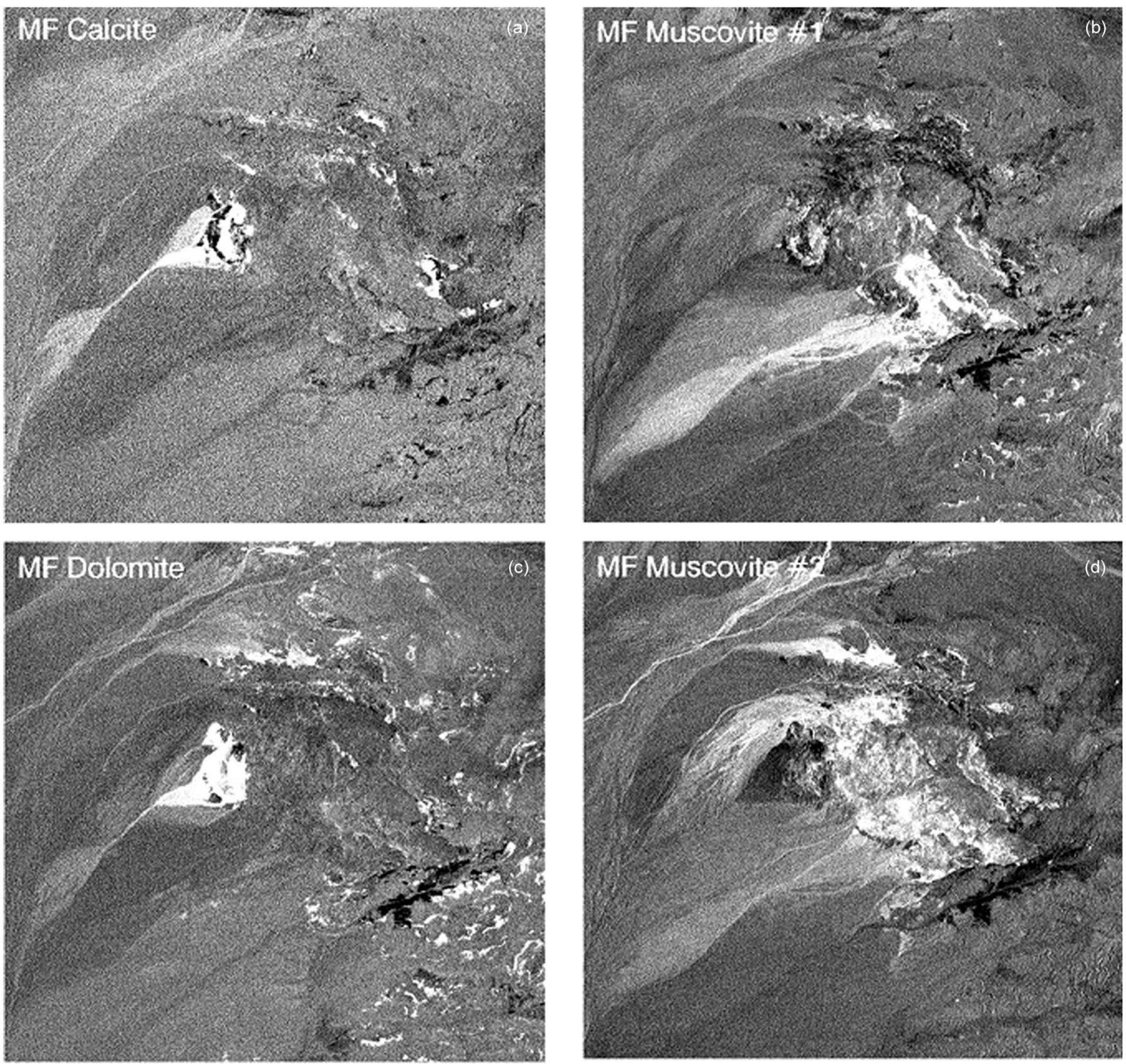

Fig. 11. MTMF results for the NDV AVIRIS data (short-wave infrared (SWIR); 2.0-2.5 $\mu \mathrm{m}$ ). The grayscale images show the MF portion of the MTMF. (a) Calcite. (b) Muscovite \#1. (c) Dolomite. (d) Muscovite \#2. The brighter pixels represent higher abundances. Note that there is potentially a significant confusion between the different minerals using MF only. See Fig. 12 for the scalebar.

data were analyzed using the previously described spectral hourglass approach. Data were corrected to reflectance using an atmospheric model, as described previously. Endmember spectra were determined by utilizing the $n$-dimensional approaches, and the locations and abundances were mapped using MTMF. The mineral signatures extracted from the data included calcite, dolomite, three varieties of muscovite, silica, and zeolites (Fig. 10).

The individual endmember abundance (MF) images (Figs. 11 and 12) were derived using the MTMF procedure and the endmembers in Fig. 10. Of note, the AVIRIS mineral abundance maps clearly show the NW-trending alteration system (muscovite \#1 and the separation of calcite and dolomite in the Precambrian roof pendant. We also used 2-D scatterplotting as described in the previous example to extract the best MTMF matches and combined these into a classification map that unambiguously shows the spectrally predominant mineral for each image pixel. Fig. 12(d) shows the combined mineral map of the spectrally predominate mineral selected using the MF abundance map in conjunction with the infeasibility images for each endmember. The mineral identifications and mapped distributions are consistent with previous mapping results using other sensors and are verified by spot checking of surface mineralogy using field reconnaissance, field and laboratory spectral measurements, and X-ray diffraction of selected hand samples [3], [38], [41]. Based on all available information, we believe that the AVIRIS MTMF mineral map itself is the best mineral map available for the site [2], [52]. 

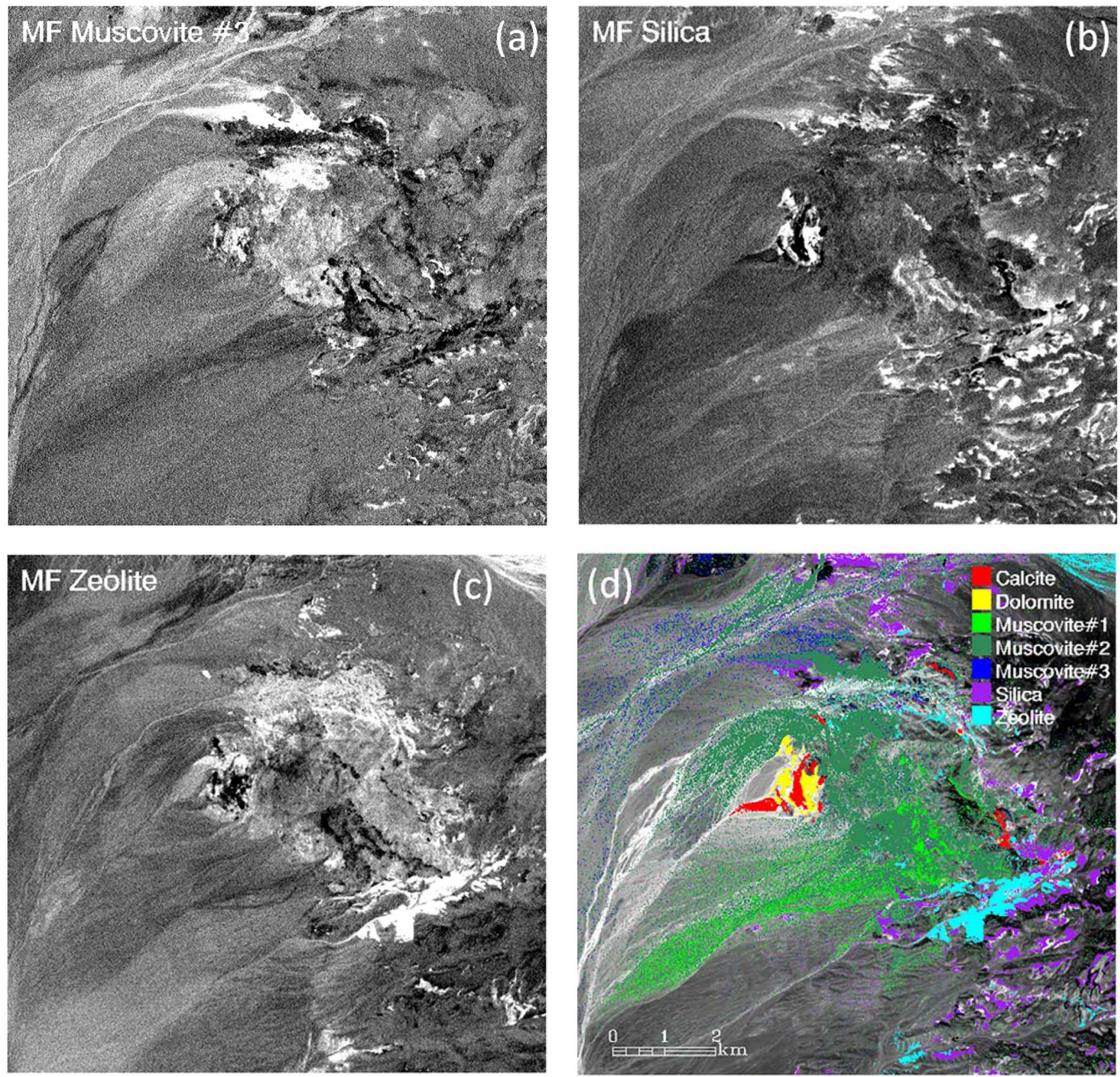

Fig. 12. MTMF results for the NDV AVIRIS data (SWIR; 2.0-2.5 $\mu \mathrm{m}$ ). The grayscale images show the MF portion of the MTMF. (a) Muscovite \#3. (b) Silica. (c) Zeolite. (d) Combined MTMF mineral map. The brighter pixels represent higher abundances. Note that there is potentially a significant confusion between the different minerals using MF only. The color classification image shows the results of scatterplotting the MF versus MT infeasibility for each endmember and selecting only those areas with high MF score and low MT infeasibility score. This results in a highly accurate mineral map [3], [38].

\section{Summary AND CONCLUSION}

Imaging spectrometry (simultaneous measurement of continuous spectra and images in up to hundreds of spectral channels or bands) is a highly evolved method for detecting, identifying, and mapping materials based on the fully resolved molecular spectral signatures. Regardless of spatial resolution, however, natural surfaces are rarely composed of a single uniform material. Analysis of imaging spectrometers therefore requires that the issue of mixed pixels be addressed. At the macroscopic scale, spectral mixing is a linear process, caused by the insensor integration of radiance from a finite pixel size. Linear spectral unmixing consists of determining the mixing endmem- bers and then estimating their abundances. An analysis process has been developed that finds endmembers in the imaging spectrometer data by taking advantage of their $n$-dimensional characteristics utilizing a convex geometry approach. Imaging spectrometer radiance data are converted to reflectance using empirical or model-based methods. The reflectance data are cast as a scattering of points in an $n$-dimensional Euclidean space, where $n$ is the number of spectral channels and all axes of the $n$-space are mutually orthogonal. Every pixel in the data set then has a point associated with it in the $n-d$ space (its Cartesian coordinates defined by the values in each spectral channel). Extreme pixels (vertices) in $n$-dimensional space correspond to 
the endmembers. Interactive $N$-dimensional visualization utilizing convex geometry concepts permits human interpretation of all spectral information of all image pixels simultaneously and projection of the endmembers back to their locations in the imagery and to their spectral signatures. Once the endmembers have been located and identified, then they can be used for the unmixing process. MF, which is a signal processing approach, has been applied to imaging spectrometer data to detect known spectral signatures in the presence of a mixed and unknown background. Unfortunately, this approach has been shown to be relatively nonselective for optical remote sensing, resulting in numerous false alarms for lower abundances. The MTMF approach was developed to mitigate this problem by combining the MF with the feasibility constraints of linear spectral mixing models. Once specific spectral endmembers are defined, partial unmixing utilizing the MTMF is used to accurately map the apparent abundance of a known target material in the presence of a background composed of many unknown and spatially varying components. MTMF incorporates the best attributes of MF but extends that technique using the linear mixed-pixel model, thus leading to high selectivity between similar materials and minimizing classification and mapping errors. We have illustrated the application of MTMF using two case histories. The analysis of the Forest Radiance HYDICE data set utilizing MF alone results in numerous false alarms for similar materials. Adding the linear mixture parameter inherent in MTMF removes false alarms by constraining the results to feasible mixtures between the background and the targets.

The use of MTMF for an AVIRIS data set of a site in NDV, $\mathrm{CA}$, produces results that are consistent with previous imaging spectrometer and field analyses but illustrating that the MTMF method provides high performance for subpixel detection of specific spectral signatures. It gains its advantage through a combined leveraging of statistical and unmixing techniques. MTMF exhibits both high degree of subpixel detection capability and outstanding false alarm rejection properties.

While the methods explained here can be used to clearly illustrate the power of imaging spectrometry data to find image endmembers and to unmix their apparent abundances on a perpixel basis, much remains to be done in this area. Endmember derivation for both the linear and nonlinear mixing problems remains a generally unsolved problem and an ill-posed one in some cases. Accurate determination of abundance fractions and validation of the results with both natural and contrived experiments are an ongoing effort. This paper has shed light on the inner workings of several popular techniques developed by the authors and in wide use in the remote sensing community.

\section{REFERENCES}

[1] A. F. H. Goetz, G. Vane, J. E. Solomon, and B. N. Rock, "Imaging spectrometry for Earth remote sensing," Science, vol. 228, no. 4704, pp. 11471153, Jun. 1985.

[2] F. A. Kruse, J. W. Boardman, and J. F. Huntington, "Evaluation and validation of EO-1 hyperion for mineral mapping," IEEE Trans. Geosci. Remote Sens., vol. 41, no. 6, pp. 1388-1400, Jun. 2003.

[3] F. A. Kruse and S. L. Perry, "Improving multispectral mapping by spectral modeling with hyperspectral signatures," J. Appl. Remote Sens., vol. 3, Jan. 2009.
[4] M. K. Hamilton, C. O. Davis, W. J. Rhea, S. H. Pilorz, and K. L. Carder, "Estimating chlorophyll content and bathymetry of Lake Tahoe using AVIRIS data," Remote Sens. Environ., vol. 44, no. 2/3, pp. 217-230, May/Jun. 1993.

[5] D. A. Roberts, M. O. Smith, and J. B. Adams, "Green vegetation, nonphotosynthetic vegetation, and soils in AVIRIS data," Remote Sens. Environ., vol. 44, no. 2/3, pp. 255-269, May/Jun. 1993.

[6] G. P. Asner and R. O. Green, "Imaging spectroscopy measures desertification in the Southwest U.S. and Argentina," Eos. Trans. AGU, vol. 80, pp. 601-605, 2001.

[7] S. Ungar, J. Pearlman, J. Mendenhall, and D. Reuter, "Overview of the Earth Observing One (EO-1) mission," IEEE Trans. Geosci. Remote Sens., vol. 41, no. 6, pp. 1149-1159, Jun. 2003.

[8] J. V. Taranik and Z. L. Aslett, "Development of hyperspectral imaging for mineral exploration," Rev. Econ. Geology, vol. 16, pp. 83-95, 2009.

[9] R. F. Kokaly, D. G. Despain, R. N. Clark, and K. E. Livo, "Spectral analysis of absorption features for mapping vegetation cover and microbial communities in Yellowstone National Park using AVIRIS data," in Integrated Geoscience Studies in the Greater Yellowstone Area-Volcanic, Tectonic, and Hydrothermal Processes in the Yellowstone Geoecosystem. Reston, VA: U.S. Geological Survey, 2007, ch. N, pp. 463-499, USGS Professional Paper 1717

[10] A. W. Nolin and J. Dozier, "Estimating snow grain size using AVIRIS data," Remote Sens. Environ., vol. 44, no. 2/3, pp. 231-238, 1993.

[11] J. W. Boardman, "Sedimentary facies analysis using imaging spectrometry: A geophysical inverse problem," Unpublished Ph.D. dissertation, Univ. Colorado, Boulder, CO, 1991, 212 p.

[12] J. B. Adams, M. O. Smith, and A. R. Gillispie, "Imaging spectroscopy: Interpretations based on spectral mixture analysis," in Remote Geochemical Analysis: Elemental and Mineralogical Composition, C. M. Pieters and P. A. Englert, Eds. Cambridge, U.K.: Univ. Cambridge, 1993, pp. $145-166$.

[13] N. Keshava and J. F. Mustard, "Spectral unmixing," IEEE Signal Process. Mag., vol. 19, no. 1, pp. 44-57, Jan. 2002.

[14] R. B. Singer and T. B. McCord, "Mars: Large scale mixing of bright and dark surface materials and implications for analysis of spectral reflectance," in Proc. 10th Lunar Planet. Sci. Conf., 1979, pp. 1835-1848.

[15] E. B. Nash and J. E. Conel, "Spectral reflectance systematics for mixtures of powdered hypersthene, labradorite, and ilmenite," J. Geophys. Res., vol. 79, no. 11, pp. 1615-1621, 1974.

[16] R. B. Singer, "Near-infrared spectral reflectance of mineral mixtures: Systematic combinations of pyroxenes, olivine, and iron oxides," J. Geophys. Res., vol. 86, no. B9, pp. 7967-7982, 1981.

[17] D. Heinz and C. Chang, "Fully constrained least squares linear spectral mixture analysis method for material quantification in hyperspectral imagery," IEEE Trans. Geosci. Remote Sens., vol. 39, no. 3, pp. 529-545, Mar. 2001.

[18] C. I. Chang, S. S. Chiang, J. A. Smith, and I. W. Ginsberg, "Linear spectral random mixture analysis for hyperspectral imagery," IEEE Trans. Geosci. Remote Sens., vol. 40, no. 2, pp. 375-392, Feb. 2002.

[19] A. Plaza, P. Martínez, R. Pérez, and J. Plaza, "A quantitative and comparative analysis of endmember extraction algorithms from hyperspectral data," IEEE Trans. Geosci. Remote Sens., vol. 42, no. 3, pp. 650-663, Mar. 2004.

[20] C. Kwan, B. Ayhan, G. Chen, J. Wang, B. Ji, and C.-I. Chang, "A novel approach for spectral unmixing, classification, and concentration estimation of chemicals and biological agents," IEEE Trans. Geosci. Remote Sens., vol. 44, no. 2, pp. 409-419, Feb. 2006

[21] L. Miao and H. Qi, "Endmember extraction from highly mixed data using minimum volume constrained nonnegative matrix factorization," IEEE Trans. Geosci. Remote Sens., vol. 45, no. 3, pp. 765-777, Mar. 2007.

[22] B. Somers, S. Delalieux, W. W. Verstraeten, J. VerBesselt, S. Lhermitte, and P. Coppin, "Magnitude- and shape-related feature integration in hyperspectral mixture analysis to monitor weeds in citrus orchards," IEEE Trans. Geosci. Remote Sens., vol. 47, no. 11, pp. 3630-3642, Nov. 2009.

[23] C.-I. Chang, W. Xiong, W. Liu, M.-L. Chang, C.-C. Wu, and C. C.-C. Chen, "Linear spectral mixture analysis based approaches to estimation of virtual dimensionality in hyperspectral imagery," IEEE Trans. Geosci. Remote Sens., vol. 48, no. 11, pp. 3960-3979, Nov. 2010.

[24] T. Kasetkasem, M. K. Arora, P. K. Varshney, and V. Areekul, "Improving subpixel classification by incorporating prior information in linear mixture models," IEEE Trans. Geosci. Remote Sens., vol. 49, no. 3, pp. 10011013, Mar. 2011

[25] J. W. Boardman, "Inversion of imaging spectrometry data using singular value decomposition," in Proc. IGARSS, 12th Can. Symp. Remote Sens., 1989, vol. 4, pp. 2069-2072. 
[26] F. A. Kruse, A. B. Lefkoff, J. B. Boardman, K. B. Heidebrecht, A. T. Shapiro, P. J. Barloon, and A. F. H. Goetz, "The Spectral Image Processing System (SIPS) - Interactive visualization and analysis of imaging spectrometer data," Remote Sens. Environ., vol. 44, no. 2/3, pp. 144-163, May/Jun. 1993.

[27] J. W. Boardman and F. A. Kruse, "Automated spectral analysis: A geological example using AVIRIS data, northern Grapevine Mountains, Nevada," in Proc. 10th Thematic Conf., Geologic Remote Sens., 1994, pp. I-407-I-418.

[28] J. W. Boardman, F. A. Kruse, and R. O. Green, "Mapping target signatures via partial unmixing of AVIRIS data," in Proc. Summ. 5th Annu. JPL Airborne Earth Sci. Workshop, 1995, pp. 23-26.

[29] D. A. Roberts, Y. Yamaguchi, and R. J. P. Lyon, "Calibration of airborne imaging spectrometer data to percent reflectance using field spectral measurements," in Proc. 19th Int. Symp. Remote Sens. Environ., Ann Arbor, MI, Oct. 21-25, 1985

[30] F. A. Kruse, "Comparison of ATREM, ACORN, and FLAASH atmospheric corrections using low-altitude AVIRIS data of Boulder, Colorado," in Proc. 13th JPL Airborne Geosci. Workshop, Pasadena, CA, Mar. 31-Apr. 2, 2004, JPL Publication 05-3.

[31] A. A. Green, M. Berman, P. Switzer, and M. D. Craig, "A transformation for ordering multispectral data in terms of image quality with implications for noise removal," IEEE Trans. Geosci. Remote Sens., vol. 26, no. 1, pp. 65-74, Jan. 1988.

[32] ENVI User's Guide, ITTVIS, Boulder, CO, 2010, unpaginated (installation) CD-ROM.

[33] J. W. Boardman, "Automated spectral unmixing of AVIRIS data using convex geometry concepts," in Proc. Summ. 4th JPL Airborne Geosci. Workshop, 1993, vol. 1, pp. 11-14, JPL Publication 93-26.

[34] A. A. Green and M. D. Craig, "Analysis of aircraft spectrometer data with logarithmic residuals," in Proc. Airborne Imaging Spectrometer Data Anal. Workshop, G. Vane and A. Goetz, Eds., Apr. 1985, pp. 111-119, JPL Publication 86-35

[35] F. A. Kruse, G. L. Raines, and K. Watson, "Analytical techniques for extracting geologic information from multichannel airborne spectroradiometer and airborne imaging spectrometer data," in Proc. Int. Symp. Remote Sens. Environ., 4th Thematic Conf. Remote Sens. Exploration Geology, 1985, pp. 309-324.

[36] Y. Yamaguchi and R. J. P. Lyon, "Identification of clay minerals by feature coding of near-infrared spectra," in Proc. Int. Symp. Remote Sens. Environ., 5th Thematic Conf. Remote Sens. Exploration Geology, Reno, NV, Sep. 29-Oct. 2, 1986, pp. 627-636.

[37] R. N. Clark, T. V. V. King, and N. S. Gorelick, "Automatic continuum analysis of reflectance spectra," in Proc. 3rd AIS Workshop, Jun. 2-4, 1987, pp. 138-142, JPL Publication 87-30.

[38] F. A. Kruse, "Use of airborne imaging spectrometer data to map minerals associated with hydrothermally altered rocks in the northern Grapevine Mountains, Nevada and California," Remote Sens. Environ., vol. 24, no. 1, pp. 31-51, Feb. 1988

[39] R. N. Clark, G. A. Swayze, A. Gallagher, N. Gorelick, and F. A. Kruse, "Mapping with imaging spectrometer data using the complete band shape least-squares algorithm simultaneously fit to multiple spectral features from multiple materials," in Proc. 3rd Airborne Visible/Infrared Imaging Spectrometer (AVIRIS) Workshop, 1991, pp. 2-3, JPL Publication 91-28.

[40] F. A. Kruse and A. B. Lefkoff, "Knowledge-based geologic mapping with imaging spectrometers," Remote Sens. Rev., vol. 8, pp. 3-28, 1993.

[41] F. A. Kruse, A. B. Lefkoff, and J. B. Dietz, "Expert system-based mineral mapping in northern Death Valley, California/Nevada using the Airborne Visible/Infrared Imaging Spectrometer (AVIRIS)," Remote Sens. Environ., vol. 44, no. 2/3, pp. 309-336, May/Jun. 1993.

[42] R. N. Clark, G. A. Swayze, K. E. Livo, R. F. Kokaly, S. J. Sutley, J. B. Dalton, R. R. McDougal, and C. A. Gent, "Imaging spectroscopy: Earth and planetary remote sensing with the USGS Tetracorder and expert systems," J. Geophys. Res., vol. 108, no. E12, pp. 5-1-5-44, 2003.

[43] F. A. Kruse, "Expert system analysis of hyperspectral data," in Proc. SPIE Defense Security, Algorithms Technol. Multispectral, Hyperspectral, Ultraspectral Imagery XIV, 2008, vol. 6966.

[44] D. O. North, "An analysis of the factors which determine signal/noise discrimination in pulsed carrier systems," RCA Labs., Princeton, NJ, Rep. PTR-6C, 1943.
[45] D. O. North, "An analysis of the factors which determine signal/noise discrimination in pulsed-carrier systems," Proc. IEEE, vol. 51, no. 7, pp. 1016-1027, Jul. 1963.

[46] J. Y. Chen and I. S. Reed, "A detection algorithm for optical targets in clutter," IEEE Trans. Aerosp. Electron. Syst., vol. AES-23, no. 1, pp. 4659, Jan. 1987.

[47] J. C. Harsanyi and C. I. Chang, "Hyperspectral image classification and dimensionality reduction: An orthogonal subspace projection approach," IEEE Trans. Geosci. Remote Sens., vol. 32, no. 4, pp. 779-785, Jul. 1994.

[48] J. W. Boardman, "Leveraging the high dimensionality of AVIRIS data for improved sub-pixel target unmixing and rejection of false positives: Mixture tuned matched filtering," in Proc. Summ. 7th Annu. JPL Airborne Geosci. Workshop, 1998, p. 55.

[49] W. S. Press, S. A. Teukolsky, W. T. Vetterling, and B. P. Flannery, Numerical Recipes: The Art of Scientific Computing, 3rd ed. New York: Cambridge Univ. Press, 2007.

[50] R. W. Basedow, D. C. Carmer, and M. E. Anderson, "HYDICE system: Implementation and performance," Proc. SPIE, vol. 2480, pp. 258-267, 1995.

[51] M. L. Nischan, J. P. Kerekes, J. E. Baum, and R. W. Basedow, "Analysis of HYDICE noise characteristics and their impact on subpixel object detection," in Proc. SPIE Conf. Imaging Spectrometry V, 1999, vol. 3753, pp. 112-123.

[52] F. A. Kruse, J. W. Boardman, and J. F. Huntington, "Fifteen years of hyperspectral data: Northern Grapevine Mountains, Nevada," in Proc. 8th JPL Airborne Earth Sci. Workshop, 1999, pp. 247-258.

[53] R. O. Green, M. L. Eastwood, and C. M. Sarture, "Imaging spectroscopy and the Airborne Visible Infrared Imaging Spectrometer (AVIRIS)," Remote Sens. Environ., vol. 65, no. 3, pp. 227-248, Sep. 1998.

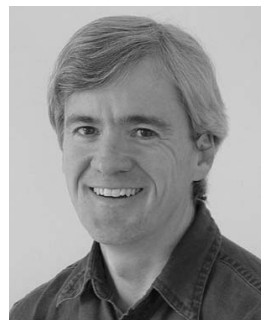

Joseph W. Boardman received the Ph.D. degree in geophysics from the University of Colorado, Boulder, Colorado, in 1991.

After his Ph.D. studies, he had a two-year postdoctoral research fellowship with the CSIRO, Sydney, Australia. From 1993 to 1995, he was a Research Associate with CSES, University of Colorado, Boulder. Since 1995, he has been a Senior Geophysicist with Analytical Imaging and Geophysics, LLC, Boulder. $\mathrm{He}$ has conducted sponsored research with a variety of commercial and government organizations, developing and applying spectral mixture analysis and photogrammetric methods to imaging spectrometer data, including AVIRIS, HyMap, Hyperion, Moon Mineral Mapper (M3), and ARTEMIS. He is also one of the scientists that originally developed the image analysis software "ENVI."

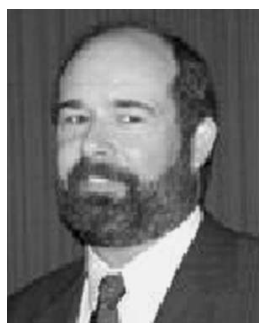

Fred A. Kruse (M'96) received the Ph.D. degree in geology from the Colorado School of Mines, Golden, in 1987.

$\mathrm{He}$ is currently a Research Professor with the Department of Physics and Remote Sensing Center, Naval Postgraduate School, Monterey, CA. He teaches courses in spectral remote sensing and synthetic aperture radar (SAR) and is developing Internet- and computer-media-based remote sensing curricula. His research focuses on physics-based exploration of diverse visible/near infrared, shortwave infrared, and long-wave infrared imaging spectrometer data and multifrequency, polarimetric, and interferometric SAR remote sensing data sets. His interests include extraction of information applicable to scientific problems in geology (geologic mapping, exploration for ore deposits, geothermal/ hydrothermal systems, geologic hazards, and environmental monitoring) and for military purposes. He is also one of the scientists that originally developed the image analysis software "ENVI." 